\title{
Capacidad de absorción de la inflación y su efecto sobre el precio de las acciones: una revisión de la literatura
}

\section{CARLOS CANO ${ }^{a}$, FRANCISCO JAREÑO ${ }^{b}$}

\begin{abstract}
RESUMEN Este artículo aborda la capacidad de absorción de la inflación que tienen las empresas clasificadas por sectores, así como su impacto en el precio de las acciones. Se comienza justificando la importancia del tema objeto de estudio, para luego presentar una revisión de la literatura internacional. Se destacan los trabajos pioneros en la materia y se aporta una clasificación de los estudios examinados. Luego, se analiza el caso español, para finalizar con las principales conclusiones del estudio. Según los autores, la capacidad de absorción de la inflación permite conocer la habilidad que tienen las empresas de un determinado sector para trasladar a precios los cambios inflacionistas que acontecen en la economía. Las empresas con alta capacidad de absorción de la inflación tenderán a presentar cotizaciones más elevadas y, además, serán menos sensibles a cambios inflacionistas.
\end{abstract}

PALABRAS CLAVE análisis sectorial, capacidad de absorción de la inflación, duración de activos, expectativas de inflación, tipos de interés nominales.

\section{HISTORIA DEL ARTÍCULO}

¿CÓMO CITAR?:

Cano, C. \& Jareño, F. (2015). Capacidad de absorción de la inflación y su efecto sobre el precio de las acciones: una revisión de la literatura. Perspectiva Empresarial, 2(1), 75-84. http://dx.doi. org/10.16967\%2Frpe.v2n1a5

RECIBIDO: 5 de agosto de 2013 APROBADO: 17 de julio de 2014

CORRESPONDENCIA:

Francisco Jareño, Universidad de Castilla-La Mancha, Plaza de la Universidad, 1, 02071 , Albacete, España.

a Máster en Consultoría y Asesoría Financiera y Fiscal, investigador del Departamento de Análisis Económico y Finanzas, Universidad de Castilla-La Mancha, España. Correo electrónico: carloscanodelolmo@hotmail.com

b Doctor en Economía Financiera y Contabilidad, profesor titular de universidad del Departamento de Análisis Económico y Finanzas, Universidad de Castilla-La Mancha, España.Correo electrónico: Francisco.Jareno@uclm.es 


\section{Inflation absorption capability and its effect on the price of shares: A review of its literature}

ABSTRACT This article addresses the inflation absorption capability of companies classified by sectors, as well as its impact in the price of shares. It starts by justifying the importance of the subject of study and then it presents a review of the international literature on the subject. The pioneer works on the subject are highlighted and a classification of the studies that have been analyzed is provided. Then, it analyzes the Spanish case in order to complete the main conclusions of the study. According to the authors, the inflation absorption capability, allows us to know the capability that companies from a determined sector have to translate the inflationary changes that happen in the economy into prices. The companies with a high capability of absorption will tend to present higher quotations, and will be less sensitive to inflationary changes.

\section{¿CÓMO CITO EL ARTÍCULO?} HOW TO CITE THIS PAPER?

\section{CHICAGO:}

Cano, Carlos y Jareño, Francisco. 2015. "Capacidad de absorción de la inflación y su efecto sobre el precio de las acciones: una revisión de la literatura". Perspectiva Empresarial 2: 75-84. http:// dx.doi.org/10.16967\%2Frpe. v2n1a5

MLA:

Cano, Carlos y Jareño, Francisco. "Capacidad de absorción de la inflación y su efecto sobre el precio de las acciones: una revisión de la literatura". Perspectiva Empresarial 2.1 (2015): 7584. Digital. http://dx.doi. org/10.16967\%2Frpe.v2n1a5
KEYWORDS sectorial analysis, inflation absorption capability, duration of assets, inflation expectation, types of nominal interest rate.

\section{Capacidade de absorção da inflação e seu efeito sobre o preço das ações: uma revisão da literatura}

RESUMO Este artigo aborda a capacidade de absorção da inflação que têm as empresas classificadas por setores, assim como seu impacto no preço das ações. Começa-se justificando a importância do tema objeto de estudo, para logo apresentar uma revisão da literatura internacional. Destacam-se os trabalhos pioneiros na matéria e fornece uma classificação dos estudos analisados. Logo, analisa-se o caso espanhol, para finalizar com as principais conclusões do estudo. Segundo os autores, a capacidade de absorção da inflação permite conhecer a habilidade que têm as empresas de um determinado setor para transladar a preços as mudanças inflacionárias que acontecem na economia. As empresas com alta capacidade de absorção da inflação tenderão a apresentar cotizações mais elevadas e, além disso, serão menos sensíveis a mudanças inflacionárias.

PALAVRAS CHAVE análise setorial, capacidade de absorção da inflação, duração de ativos, expectativas de inflação, taxa de juros nominal. 


\section{Introducción}

El presente trabajo tiene como principal objetivo la revisión del concepto que presentamos como estimación de la capacidad que poseen las empresas para transmitir los cambios inflacionistas existentes en la economía, a los precios de los productos que venden o de los servicios que prestan, mediante la aplicación de las técnicas econométricas pertinentes. Principalmente analizamos el efecto que dicha capacidad de absorción de la inflación tiene sobre los precios de las acciones de las compañías que cotizan en el mercado bursátil. De este modo, podremos evaluar los resultados obtenidos en esta línea con los trabajos existentes a nivel internacional, así como para el caso español.

En este sentido, se realiza una profunda revisión de la literatura existente en este ámbito, destacando los trabajos de Asikoglu \& Ercan (1992) y Jareño \& Navarro (2010) como base para nuestro análisis. Asimismo, este estudio aporta una clasificación de los principales trabajos revisados y recoge las principales conclusiones extraídas, producto del análisis de los mismos.

\section{Revisión de literatura previa internacional}

En relación con la capacidad de las empresas de repercutir a precios las variaciones inflacionistas de la economía, hay una gran diversidad de literatura previa que vamos a revisar en primera instancia, de cara a sentar las bases de nuestro trabajo. De esta manera, se observará la evolución de los estudios que se han realizado hasta la fecha, aplicados a distintos ámbitos geográficos, y así podremos desarrollar un trabajo con matices innovadores.

Con carácter previo a dicha revisión, hemos de decir que la base fundamental sobre la que se va a asentar este estudio está formada por los trabajos de Asikoglu \& Ercan (1992) para el caso estadounidense, y para el caso español, Jareño (2006a) y Jareño \& Navarro (2010), si bien es cierto que la denominación de capacidad de absorción de la inflación fue introducida por primera vez por Estep \& Hanson (1980), a cuyos análisis se hace referencia en la bibliografía previamente citada.

Comenzaremos haciendo alusión a Asikoglu \& Ercan (1992), quienes a partir de numerosas investigaciones analizaron la relación negativa entre la inflación y los rendimientos de las acciones: la hipótesis de la dependencia financiera de Lintner (1975), la hipótesis del sistema de tributación de Feldstein (1980), la hipótesis de Fama (1981 y 1982), etc.

En el sector financiero, se puede afirmar que un incremento en los tipos de interés hace que el valor actual de los activos bancarios caiga más que sus pasivos u obligaciones. Además, los costes de esos pasivos aumentan más rápidamente que la rentabilidad de los activos bancarios. Este hecho confirma la relación negativa entre un cambio en la inflación y la cotización de una acción.

En el resto de empresas no financieras, los movimientos en los tipos de interés tienen un efecto significativo sobre el valor de dichas empresas (no financieras) a través de diferentes canales. En primer lugar, en el marco de los modelos de valor actual, incrementos en los tipos de interés aumentan el coste de capital de las empresas, lo que se traduce en mayores tasas de descuento para la valoración de futuros flujos de efectivo, lo que afecta negativamente a las cotizaciones bursátiles de las empresas. En segundo lugar, subidas de tipos de interés aumentan el gasto por intereses de las empresas apalancadas o endeudadas, y también pueden reducir la demanda de productos por parte de los consumidores más endeudados. Este hecho provoca una rentabilidad de las empresas más baja, y tiene un impacto negativo en los precios de las acciones. En tercer lugar, los cambios en los tipos de interés modifican el valor de mercado de los activos y pasivos financieros mantenidos por las sociedades no financieras. Además, las fluctuaciones de los tipos de interés afectan el coste de oportunidad de las inversiones de renta variable. Los tipos de interés más altos hacen que los bonos sean más atractivos como una alternativa al mantenimiento de acciones, lo que lleva a los inversores a ajustar sus carteras mediante la compra de bonos y venta de acciones, bajando de este modo los precios de las acciones.

Todos estos efectos sugieren una relación inversa entre los movimientos de los tipos de interés y los rendimientos de las acciones.

En este contexto, Asikoglu \& Ercan (1992) estudian la mencionada relación entre inflación y rendimientos de acciones a nivel sectorial en Estados Unidos, utilizando la hipótesis constante de la capacidad de repercutir a precios los cambios en la inflación (tomada de Estep y Hanson en su estudio de 1980). Concretamente, el efecto negativo de un incremento de inflación sobre el 
precio de cotización de una empresa estará inversamente relacionado con su capacidad para repercutir a precios la variación de la inflación.

Así, la evidencia empírica que se presenta sugiere un efecto de la capacidad de absorción de la inflación sobre la valoración de las acciones, demostrándose además que los coeficientes que la miden en los distintos sectores difieren sustancialmente. En esta línea, en las industrias en las que dichos coeficientes son mayores, los precios de las acciones o títulos de renta variable son menos sensibles a los cambios inflacionistas, e incrementos de la capacidad de absorción de la inflación están asociados a precios más altos por acción.

Se parte del modelo desarrollado por Estep \& Hanson (1980), quienes toman como base el modelo de descuento de dividendos (MDD). Concretamente, la expresión de Gordon-Shapiro asume que los dividendos futuros están determinados por una tasa de crecimiento constante, lo cual conduce a la conocida fórmula de valoración de las acciones (Gordon, 1959; Gordon \& Shapiro, 1956):

$$
P=\sum_{t=1}^{\infty} \frac{D_{0}(1+g)^{t}}{(1+k)^{t}}=\frac{D_{0}(1+g)}{k-g}
$$

donde $P$ equivale al valor teórico de las acciones, $D_{0}$ se corresponde con el primer dividendo pagado por la empresa, g es la tasa de crecimiento esperada de los dividendos y $k$, la tasa de descuento nominal.

Adicionalmente, otro aspecto que hemos de tener presente de cara a esta valoración de las acciones, al igual que hacían Leibowitz \& Kogelman (2000), es la difusión del factor franquicia ${ }^{1}$, es decir, la consideración de las ganancias incrementales que se generan en la empresa en cuestión, como consecuencia de los análisis/investigaciones que esta lleve a cabo sobre el coste del capital. La inclusión de este nuevo factor 1) clarifica el papel del $\mathrm{ROE}^{2}, 2$ ) facilita el desarrollo de modelos de dos fases que reflejan el patrón típico de

1 Según Leibowitz y Kogelman (1990 y 1993) y Jareño (2005), el valor teórico de una acción se puede dividir en dos componentes: valor tangible (asociado a la actividad actual de la empresa) y valor franquicia (asociado a las oportunidades de inversión y crecimiento futuro o "nueva actividad").

2 Return On Equity: se trata de un ratio que evalúa la rentabilidad de los fondos propios de la empresa. las ganancias de la compañía (corto y largo plazo), 3) apoya diversos niveles del $\mathrm{PER}^{3}$, solventando problemas de artificialidad cuando este toma valores altos, siendo forzado con estimaciones bajas para la prima de riesgo o la tasa de inflación, y 4) conduce a una expresión de la duración de las acciones que es igual al PER en ciertas condiciones de estabilidad. Algunos de los conceptos aplicados en este trabajo procedían de estudios previos de dichos autores, como Leibowitz \& Kogelman (1990, 1991 y 1993).

Retomando el trabajo de Estep \& Hanson (1980), hemos de referirnos a la ampliación del modelo tradicional de descuento de dividendos que ellos hicieron para incorporar el efecto diferencial de la inflación sobre el crecimiento de los dividendos. No obstante, la presencia de la inflación requiere matizar dos aspectos:

1. En primer lugar, la tasa de rendimiento requerida en las inversiones se ajusta con la inflación, de acuerdo con la relación de Fisher, según la cual el tipo de interés nominal (k) en cualquier periodo es, en su versión aproximada, igual a la suma del tipo de interés real (R) más la tasa de inflación esperada (I).

$$
(1+\mathrm{k})=(1+\mathrm{R})(1+\mathrm{I})
$$

En relación con el efecto Fisher, Jareño \& Tolentino (2012b) lo definen como el porcentaje en el que los tipos de interés nominales contienen expectativas de inflación, manteniendo constante el tipo de interés real. Por tanto, cuando la tasa de inflación esperada aumenta en un punto porcentual $\left(\pi_{t}^{e}\right)$, el tipo de interés nominal $\left(i_{t}\right)$ también aumenta en el mismo porcentaje, si el tipo de interés real $\left(r_{t}^{e}\right)$ se mantiene constante. Matemáticamente, la ecuación de Fisher se puede descomponer de la siguiente manera (sin oportunidades de arbitraje):

$$
\begin{aligned}
& \left(1+i_{t}\right)=\left(1+r_{t}^{e}\right) *\left(1+\pi_{t}^{e}\right) \rightarrow 1+i_{t}=1+r_{t}^{e}+\pi_{t}^{e} \\
& +\left(r_{t}^{e} * \pi_{t}^{e}\right) \rightarrow i_{t}=r_{t}^{e}+\pi_{t}^{e}+\left(r_{t}^{e} * \pi_{t}^{e}\right)
\end{aligned}
$$

No obstante, el valor del producto del tipo de interés y la tasa de inflación $\left(r_{t}^{e} * \pi_{t}^{e}\right)$ generalmente es despreciable (prácticamente 0), pudiendo omitirlo en la mayoría de los casos. De este

3 Price to Earnings Ratio: es el cociente entre el precio de una acción de la empresa y el beneficio que esta otorga. 
modo, obtendríamos una expresión simplificada del efecto Fisher, acorde con lo comentado en el párrafo previo a la ecuación (2):

$$
\begin{aligned}
& i_{t} \approx r_{t}^{e}+\pi_{t}^{e} \\
& r_{t}^{e} \approx i_{t}-\pi_{t}^{e}
\end{aligned}
$$

Así, llegamos a una relación 1:1 entre $i_{t}$ y $\pi_{t}^{e}$ : un aumento en la inflación esperada conlleva un incremento proporcional en el tipo de interés nominal $\left(\Delta i_{t}=\Delta \pi_{t}^{e}\right)$.

En este sentido, dichos autores también suministran documentación bastante relevante para el caso que nos atañe. El mencionado efecto tiene implicaciones sobre la capacidad de absorción de la inflación, concretamente, sobre la sensibilidad del precio de los activos de renta variable cuando el tipo de interés nominal cambia a consecuencia de variaciones en la tasa de inflación esperada (Jareño, 2005; Jareño \& Navarro, 2010).

Así, Jareño \& Tolentino (2012b) analizan para el caso español si la mayoría de los movimientos del tipo de interés nominal son causados por la tasa de inflación, manteniendo constante el tipo de interés real. De acuerdo con la literatura previa, este efecto se da solo parcialmente y en el largo plazo, por lo que no podemos suponer que un cambio en las expectativas de inflación (ocurrido en un momento determinado), influya sobre el tipo de interés nominal de la economía de forma instantánea y total. Para el caso español se encuentra una relación estadística positiva y significativa entre las variaciones en la inflación esperada y los cambios en el tipo de interés nominal (para el periodo comprendido entre 1993 y 2004).

Además, según Jareño \& Navarro (2010), cambios en el tipo de interés nominal pueden tener diferente impacto sobre el precio de las acciones, según si es inducido por un cambio en las expectativas de inflación o en el tipo de interés real. En este contexto, si el efecto Fisher existe, una empresa capaz de transmitir un incremento general de la tasa de inflación al precio de sus productos de manera total (y, consecuentemente, a sus ganancias nominales esperadas), dejaría el precio de las acciones inalterado. Por el contrario, una baja capacidad para trasladar a precios los cambios en la inflación llevaría a una sensibilidad extremadamente alta del precio de las acciones ante cambios en el tipo de interés.

Asimismo, la capacidad de absorción de la inflación dependerá de la industria en la que se opere, de modo que si un sector o una empresa está expuesta a la competencia extranjera (dicha capacidad sería casi nula) no tendría la misma respuesta que si existe poder de mercado (donde la respuesta de los precios de las acciones ante cambios en el tipo de interés nominal, debidos a variaciones en las expectativas de inflación, será mínima).

2. En segundo lugar, el crecimiento de los dividendos corporativos se descompone en crecimiento real y en crecimiento debido a la inflación.

$$
(1+g)=(1+G)(1+f I)
$$

donde $\mathrm{G}$ representa la tasa de crecimiento real y $\mathrm{f}$ es el coeficiente de absorción de la inflación.

De este modo, la tasa de crecimiento de los dividendos está determinada por la tasa de crecimiento real de la empresa y un término que representa el crecimiento ocasionado por la inflación. Sustituyendo ambos ajustes en la ecuación (1), Estep \& Hanson (1980) obtienen el modelo de capacidad de absorción de la inflación:

$$
P=\frac{D_{0}(1+g)(1+f I)}{(1+R)(1+I)-(1+G)(1+f I)}
$$

Además, despejando f en la ecuación (6), se obtiene la fórmula final con la que calculan los coeficientes de absorción de la inflación:

$$
f=\frac{g-G}{(1+G) I}
$$

En esta línea, si una empresa es capaz de transformar toda la inflación en crecimiento futuro, su coeficiente de absorción de la inflación será unitario, y el precio real de sus acciones será independiente de la inflación. Si, en cambio, el crecimiento es fijo, el coeficiente es cero. En general, el impacto negativo de una inflación notablemente alta sobre el precio de las acciones está inversamente relacionado con el nivel del coeficiente de absorción de la inflación, y el precio de las acciones por sí mismo está relacionado positivamente con dicho coeficiente.

Autores como Estep \& Hanson (1980) y Asikoglu \& Johnson (1986) estiman la capacidad para trasladar a precios los cambios en la inflación en las industrias norteamericanas y canadienses, respectivamente. La significación estadística del efecto de la absorción de la inflación en la valoración de las acciones ha sido probada por Asikoglu 
\& Johnson (1990), usando datos de siete países industrializados en mercado agregado.

Sin embargo, Asikoglu \& Ercan (1992) van más allá estimando dichos coeficientes a nivel sectorial para 14 industrias norteamericanas en el periodo 1974-1988, siguiendo las pautas de la guía de analistas de Standard and Poor's, a la par que analizan su validez estadística mediante la estimación de la siguiente ecuación:

$$
R_{i}=\beta_{0}+\beta_{1} * I+\beta_{2}\left(D U_{i}^{*} I\right)+\beta_{3} * f_{i}+u_{i}
$$

donde $R_{i}$ es el porcentaje de cambio del precio de las acciones para la industria i, I es la tasa de inflación, $D U_{i}$ es la variable categórica específica de la industria i, y $f_{i}$ es el coeficiente de absorción de la inflación para la industria i.

La variable categórica específica de cada industria toma valor unitario si la media muestral de las estimaciones de la capacidad de absorción de la inflación para cada una de esas industrias es igual o mayor que la unidad (en caso contrario, su valor será 0).

El coeficiente $\beta_{2}$ mide el efecto diferencial de la inflación sobre el precio de las acciones tanto en industrias con alto coeficiente de absorción de la inflación (mayor que la unidad), como en aquellas que posean un coeficiente bajo (menor que la unidad). En este último caso, el precio de las acciones se verá influenciado a través del coeficiente $\beta_{1}$, por incrementos en la tasa de inflación. En cambio, si la industria posee un coeficiente de absorción de la inflación elevado, el efecto de la inflación sobre el precio de las acciones será $\beta_{1}+\beta_{2}$.

Por otro lado, el vector de observaciones de coeficientes -que miden la capacidad de trasladar a precios los cambios en la inflación- de cada sector específico $\left(f_{i}\right)$ recoge la correlación entre las variaciones de los precios de las acciones a lo largo del tiempo y los coeficientes de absorción de la inflación.

Al igual que en estudios realizados con anterioridad, se espera una relación negativa entre la tasa de inflación y el rendimiento de las acciones, de modo que el signo de $\beta_{1}$ será, a priori, negativo (al contrario que para los coeficientes $\beta_{2}$ y $\beta_{3}$ ). Asimismo, el efecto negativo de la inflación sobre el precio las acciones será menor si la industria en cuestión tiene un coeficiente de absorción de la inflación alto $\left(\beta_{2}>0\right)$. Del mismo modo, un incremento en la capacidad de absorción de la inflación de una industria a lo largo del tiempo afectará el precio de sus acciones favorablemente $\left(\beta_{3}>0\right)$.
Tras analizar los resultados de las diferentes pruebas de regresión, la evidencia empírica pone de manifiesto que, a pesar de la relación negativa ya comentada, las empresas con mayores coeficientes de absorción de la inflación son menos sensibles a la inflación que aquellas con un coeficiente bajo. Además, los precios de las acciones se benefician de los incrementos en la capacidad para trasladar a precios los cambios en la inflación a lo largo del tiempo.

En cualquier caso, se aprecian claras diferencias entre los coeficientes de absorción de la inflación para cada una de las industrias analizadas, según este sea alto o bajo. Así, se demuestra que no todas ellas tienen la misma capacidad para mantener el crecimiento de sus beneficios durante un periodo de inflación. Además, la evidencia empírica refleja la existencia de un efecto de la capacidad que presentan las empresas para trasladar a los precios de sus productos o servicios los cambios en la inflación en la valoración de las acciones en un entorno inflacionista, el cual opera de dos maneras:

- Primero, las industrias con una baja capacidad de absorción de la inflación son más sensibles a cambios en la tasa de inflación (y viceversa). Este resultado corrobora la principal idea de que, cuando crece la inflación, la presión sobre los precios de las acciones a través de la tasa de descuento será contrarrestada hasta cierto punto por incrementos en el crecimiento esperado de la rentabilidad nominal. Este efecto compensatorio está relacionado positivamente con el coeficiente de absorción de la inflación de la industria en cuestión.

- En segundo lugar, hay una relación positiva entre los cambios en los precios de las acciones y los coeficientes que miden la capacidad de la empresa para trasladar a precios los cambios en la inflación, es decir, los inversores estarían dispuestos a pagar un mayor precio cuando una mayor parte de la tasa de inflación dada repercute en las acciones en forma de crecimiento de las ganancias.

\section{El caso español}

Centrándonos en el caso español, varios son los autores que han abordado este tema, bien con carácter estimativo de los coeficientes de absorción de la inflación, o bien como factor explicativo de otros aspectos teóricos, como puede ser la 
paradoja de la duración de las acciones. Según Jareño \& Navarro (2010), dicha paradoja se define como la diferencia entre la duración teórica del activo derivada del modelo de descuento de dividendos (MDD) y su estimación empírica. Esta idea sugiere que si una empresa puede trasladar los cambios en la inflación a los precios de sus productos y, por tanto, a sus beneficios y dividendos, entonces los cambios en los tipos de interés nominales debido a cambios en la inflación esperada tendrán un impacto limitado sobre los precios de las acciones. A continuación pasamos a ver algunos de los estudios más relevantes al respecto.

Comenzamos con los trabajos de Jareño (2006b) y Jareño \& Navarro (2010), cuyo principal resultado es la obtención de una fuerte relación negativa entre sensibilidad de los rendimientos de las acciones ante cambios en los tipos de interés nominales y la capacidad de absorción de la inflación, siendo una posible explicación a la llamada paradoja de la duración de las acciones. Concretamente, dicha capacidad de absorción puede explicar alrededor de un 50\% de las diferencias encontradas entre sectores en las duraciones de las acciones ante cambios en tipos de interés nominales. Asimismo, un amplio número de empresas tiene sensibilidad significativa y negativa ante cambios en los tipos de interés reales, y negativa pero no significativa (por lo general) ante cambios en tasa de inflación esperada.

De esta manera, se contribuye al mercado español analizando la sensibilidad de los rendimientos sectoriales ante cambios en los tipos de interés reales de forma separada de la sensibilidad ante cambios en la tasa de inflación esperada. También se tienen en cuenta otros factores explicativos de las empresas, como puede ser el endeudamiento, la liquidez, el tamaño o las opciones de crecimiento.

No obstante, en el marco español también destacan trabajos como el de Ballester et al. (2011), en el cual se establece una relación permanente y negativa entre la tasa de inflación española y los rendimientos bursátiles a largo plazo. Asimismo, los mencionados autores utilizan la capacidad de absorción de la inflación como factor explicativo al hablar de la exposición de los bancos al tipo de interés.

También se utiliza la capacidad de trasladar a precios los cambios en la inflación como factor explicativo al analizar su relación con la duración de las acciones (sensibilidad de las mismas ante cambios en los tipos de interés) para las empresas españolas, tal y como podemos observar en Jareño \& Tolentino (2012a). Dicho estudio, basado en los modelos de duración propuestos por Jareño (2006b y 2008), separa las variaciones en los tipos de interés nominales derivadas de cambios en los tipos de interés reales y en la tasa de inflación esperada.

Además, al igual que en Jareño \& Navarro (2010), se muestra evidencia de la relación negativa entre la duración de la inflación y la capacidad de absorción de la inflación. Sin embargo, esta relación negativa no es definitiva, lo que corrobora los resultados obtenidos por Jareño \& Tolentino (2012b), debido a la existencia de efecto Fisher parcial en España.

Por otro lado, Díaz \& Jareño (2009) analizan, en primer lugar, la respuesta a corto plazo de los rendimientos sectoriales de las acciones ante anuncios del componente no anticipado de la inflación y, en segundo lugar, profundizan en los potenciales factores explicativos que influyen sobre la misma.

En este sentido, en el primer análisis se hace hincapié en la dirección de las sorpresas inflacionistas y en el estado de la economía, observándose una respuesta positiva y significativa de la rentabilidad de las acciones ante "malas noticias" en épocas de recesión (es decir, una tasa de inflación mayor a la esperada), y también en caso de sorpresas de inflación negativas ("buenas noticias") en fases recesivas del ciclo económico. La explicación de este comportamiento reside en que los agentes económicos pueden pensar que la economía está creciendo por encima de sus expectativas, sobre lo cual también tiene influencia la elevada capacidad que poseen las empresas para trasladar a precios los cambios en la tasa de inflación.

En el segundo estudio, el modelo de descuento de dividendos es usado como estructura teórica para controlar los principales componentes de los precios de las acciones: el tipo de interés libre de riesgo, la prima de riesgo de dichas acciones y las expectativas de crecimiento de los beneficios de la compañía. Así, los dos primeros factores no muestran respuestas significativas ante sorpresas inflacionistas inesperadas, si bien es cierto que las expectativas de crecimiento de los beneficios de la empresa pueden explicar la mayor parte del comportamiento del rendimiento de las acciones ante las noticias de inflación.

En esta línea, los resultados son coherentes con la evolución de las expectativas de crecimiento de los dividendos de la empresa, ya que 
la relación entre este componente teórico de los precios de las acciones y la inflación no esperada parece explicar la trayectoria observada. Con carácter previo, Jareño (2007 y 2009) muestra algunos de estos aspectos, así como la repercusión del cambio de metodología en el cálculo del IPC sobre la respuesta a nivel sectorial de la que venimos hablando.

Para finalizar las referencias al caso español, Díaz \& Jareño (2013) en sus trabajos preliminares afirman que las respuestas a corto plazo de los precios de las acciones ante determinadas noticias macroeconómicas podrían conllevar resultados sesgados, puesto que las respuestas obtenidas en diferentes escenarios pueden anularse entre sí. En este sentido, profundizan en las hipótesis planteadas, de modo que en el análisis de las noticias de inflación sobre el mercado de valores español, cobran especial relevancia la dirección de mercado de las mismas y el coeficiente de absorción de la inflación según el sector de actividad en que se ubique cada empresa.

De esta manera, noticias de inflación no anticipadas implican rendimientos anormales en función de la dirección de las noticias, el estado de la economía y la capacidad del sector para trasladar a precios los cambios en la inflación. Además, el impacto de sorpresas positivas de inflación afecta a la rentabilidad de muchos más sectores que en el caso de impactos negativos de la misma, especialmente en épocas de estancamiento o recesión económica. Esos efectos significativos son principalmente observados en sectores caracterizados por una capacidad de absorción de la inflación poco elevada. En este sentido, los resultados obtenidos son bastante consistentes respecto a la capacidad de cada industria para trasladar a precios los cambios en la inflación y las afirmaciones de Veronesi (1999).

\section{Otros casos particulares}

Abandonando el ámbito español, también se ha hecho revisión de literatura a nivel internacional, encontrando algunos trabajos interesantes que toman como muestra algunos países del Este. Por ejemplo, Ergun et al. (2008) analizan para el periodo 1996-2008 el impacto de repercusiones externas sobre el funcionamiento de la Bolsa de Valores de Estambul, utilizando para ello indicadores monetarios y macroeconómicos de Turquía. Los resultados obtenidos indican ausencia de efecto dólar sobre las relaciones internas de la Bolsa de Valores de Estambul, a la par que ponen de manifiesto una causalidad bilateral entre esta y el tipo de interés.

Por otro lado, Limpanithiwat \& Rungsombudpornkul (2010) examinan la relación entre la inflación y el precio de las acciones en Tailandia (en el periodo 2000-2010), considerando el impacto de eventos específicos como son el tsunami y la crisis económica. Los resultados obtenidos demuestran que las variaciones del precio de las acciones son irrelevantes respecto a la inflación, estando dicho resultado en concordancia con la opinión de los expertos sobre la Bolsa de Tailandia.

Otros estudios, como el de Ertek (2009), se basan en el concepto de capacidad FT introducido por Estep \& Hanson (1980), de modo que cuanto mayor sea esta (o, en otras palabras, cuanto mayor sea el porcentaje de los cambios en la inflación que se traduce en crecimiento de la tasa de beneficios de la compañía), acarreará mayores implicaciones positivas para la empresa (y viceversa).

En esa línea, Ertek (2009) toma la capacidad de absorción de la inflación como variable por considerar de cara a construir un modelo cuantitativo de selección de acciones, con la finalidad de crear carteras de calidad con cobertura de inflación en el ámbito europeo. Así, las mencionadas carteras se construyen mediante la selección de empresas individuales con base en un elenco de características que se cree que afectan el rendimiento obtenido por los diversos activos, el cual se entiende vinculado con la cobertura de la inflación.

Las variables que, junto a los coeficientes de absorción de la inflación, se tienen en cuenta para hacer tal selección de empresas son: la tasa de crecimiento nominal de los dividendos por acción, la tasa de crecimiento de los beneficios, el poder de mercado, el margen de beneficio bruto, el carácter cíclico propio de la realidad económica y, por último, los rendimientos obtenidos.

Después de haber construido varias carteras, estas son evaluadas en función de sus características de cobertura de la inflación y de sus prestaciones en general. Una primera prueba para ello consiste en la estimación de los coeficientes de correlación simple entre los beneficios o rendimientos derivados de las carteras y la inflación. Un segundo método se basa en regresiones "multivariantes", en las que los rendimientos de las carteras forman una regresión con la inflación y con las rentabilidades de la cartera media de mercado. Por tanto, vemos que la selección de 
TABLA 1. Resumen de la literatura previa relacionada con la capacidad de absorción de la inflación (AI)

\begin{tabular}{|c|c|c|c|}
\hline AUTORES & ÁMBITO & CAPACIDAD AI & CONTENIDO \\
\hline Estep \& Hanson (1980) & EE.UU. & Variable estimada & $\begin{array}{l}\text { Estimación capacidad Al (sectorialmente), usando } \\
\text { MDD ampliado }\end{array}$ \\
\hline Asikoglu \& Johnson (1986 y 1990) & Canadá & Variable estimada & Estimación capacidad Al (sectorialmente) \\
\hline Asikoglu \& Ercan (1992) & EE.UU. & Variable estimada & $\begin{array}{l}\text { Relación inflación-rendimiento de las acciones } \\
\text { (sectorialmente) }\end{array}$ \\
\hline Jareño (2006a) & España & Variable estimada & Estimación capacidad Al (sectorialmente) \\
\hline Ertek (2009) & Europa & Variable estimada & $\begin{array}{l}\text { Modelo de selección de acciones para crear carteras } \\
\text { de calidad de cobertura de la inflación }\end{array}$ \\
\hline $\begin{array}{l}\text { Jareño (2005, 2006b y 2008); } \\
\text { Jareño \& Navarro (2010); } \\
\text { Jareño \& Tolentino (2012a); } \\
\text { Jareño \& Tolentino (2012b) }\end{array}$ & España & Variable explicativa & $\begin{array}{l}\text { Duración de las acciones y capacidad Al (efecto } \\
\text { Fisher: separación del efecto del ti real y de la } \\
\text { inflación) }\end{array}$ \\
\hline Ballester et al. (2011) & España & Variable explicativa & $\begin{array}{l}\text { Exposición de los bancos al ti. Relación inflación y } \\
\text { rendimientos a l/p }\end{array}$ \\
\hline $\begin{array}{l}\text { Díaz \& Jareño (2009 y 2013); } \\
\text { Jareño (2007 y 2009) }\end{array}$ & España & Variable explicativa & $\begin{array}{l}\text { Relación inflación no anticipada-rendimiento de } \\
\text { acciones a c/p. Repercusiones del método de } \\
\text { elaboración del IPC y de la situación }\end{array}$ \\
\hline Ergun et al. (2008) & Turquía & Variable explicativa & $\begin{array}{l}\text { Relación cambios externos-rendimiento de la Bolsa } \\
\text { de Valores }\end{array}$ \\
\hline $\begin{array}{l}\text { Limpanithiwat \& } \\
\text { Rungsombudpornkul (2010) }\end{array}$ & Tailandia & Variable explicativa & $\begin{array}{l}\text { Relación inflación-precio de las acciones. Efecto crisis } \\
\text { y tsunami }\end{array}$ \\
\hline Ang et al. (2011) & Europa & $\begin{array}{l}\text { Nueva vía de } \\
\text { estimación }\end{array}$ & $\begin{array}{l}\text { Capacidad de cobertura de la inflación a nivel } \\
\text { individual (por acción) }\end{array}$ \\
\hline
\end{tabular}

Fuente: elaboración propia

valores no ocurre en un entorno "univariante", sino "multivariante".

Para finalizar, Ang et al. (2011) estudian la capacidad de cobertura de la inflación de las acciones individuales, ya que estas tienen una gran capacidad para ello, incluso cuando el mercado agregado tiene unas propiedades paupérrimas. No obstante, hay una gran dispersión de los factores beta de inflación a través de las acciones individuales, y resulta bastante difícil encontrar acciones que ofrezcan buena cobertura de la inflación ex-ante. En este sentido, se aporta una nueva vía de cara a medir la capacidad de absorción de la inflación.

\section{Conclusiones}

Los planteamientos expuestos en el presente trabajo tienen como principal objetivo analizar el concepto que estudia la capacidad que poseen las empresas para repercutir en los precios de sus productos o servicios prestados, los cambios en la inflación que acontecen en la economía, agrupando la información en sectores de actividad.

La literatura analizada destaca que la capacidad de absorción de la inflación que presentan las empresas difiere considerablemente según el sector en el que nos encontremos, haciendo especial hincapié en los trabajos de Asikoglu \& Ercan (1992) y Jareño \& Navarro (2010). Este resultado se debe a que cada sector se caracteriza por un elenco de peculiaridades que, generalmente, difiere del resto: nivel de competencia, estrategias de competitividad, poder de mercado, exposición a la coyuntura económica general, etc.

También se verifica una relación positiva entre la variación experimentada por las cotizaciones de las acciones de las empresas enmarcadas dentro un mismo sector y el coeficiente de absorción de la inflación correspondiente al mismo. Esto se debe a que los inversores estarán dispuestos a pagar un mayor precio por las acciones cuando una mayor parte de la tasa de inflación dada repercute sobre las mismas en forma de crecimiento de las ganancias/dividendos obtenidos. De este modo, incrementos de los coeficientes de absorción de la inflación se asocian a precios más altos por acción.

Asimismo, la evidencia empírica pone de manifiesto que en aquellas industrias en las que se goza de una mayor capacidad para repercutir a precios, las cotizaciones de las acciones son menos sensibles a las fluctuaciones inflacionistas en la mayoría de los casos. Esto se debe a la existencia de una relación negativa entre la inflación y las cotizaciones de las acciones, si bien es cierto que 
en aquellos sectores en los que la capacidad de absorción de la inflación es relativamente alta, los cambios en la inflación se trasladarán, prácticamente en su totalidad, al precio de los productos vendidos o servicios prestados. De este modo, los inversores no desconfiarán sobre el precio de las acciones, ya que la valoración de las mismas podrá mantenerse intacta.

\section{Referencias}

Ang, A., Brière, M. \& Signori, O. (2011). Inflation and individual equities. Financial Analysts Journal, 68(4), 36-55.

Asikoglu, Y. \& Ercan, M. R. (1992). Inflation Flow-through and Stock Prices. Journal of Portfolio Management, 18(3), 63-68.

Asikoglu, Y. \& Johson, L. D. (1986). Inflation and Stock Prices: Empirical Estimation of Flowthrough Constants. (Queen's University Working Paper 86-20).

Asikoglu, Y. \& Johson, L. D. (1990). Inflation and International Stock Prices. (Queen's University Working Paper, 90-26).

Ballester, L., Ferrer, R. \& González, C. (2011). Linear and nonlinear interest rate sensitivity of Spanish banks. The Spanish Review of Financial Economics, 9(2) 35-48.

Díaz, A. \& Jareño, F. (2009). Explanatory factors of the inflation news impact on stock returns by sector: the Spanish case. Research in International Business and Finance, 23(3), 349-368.

Díaz, A. \& Jareño, F. (2013). Inflation News and Stock Returns: Market Direction and Flow-Through Ability. Empirical Economics, 44(2), 775-798.

Ergun, U., Hassan, A., Mohd, S. \& Jusoh, M.B. (2008). External Shock Effect on the Internal Linkages of Istanbul Stock Exchange. European Journal of Economics, Finance and Administrative Sciences. ISSN 1450-2275 Issue 14.

Ertek, G. (2009). Stock Selection Strategies to construct inflation hedging portfolios. Tesis doctoral. Erasmus University.

Estep, T., \& Hanson, N. (1980). The Valuation of Financial Assets in Inflation. Saloinon Brothers Stock Research Paper.

Gordon, M. J. (1959). Dividends, Earnings and Stock Prices. Review of Economics and Statistics, 41(2), 99-105.

Gordon, M. J. \& Shapiro, E. (1956, October). Capital Equipment Analysis: The Required Rate of Profit. Management Science, 3(1), 102-110.

Jareño, F. (2005). Flow-through Capability: The Spanish Case. Journal of Asset Management, 6(3), 191-205.
Jareño, F. (2006a). Riesgo de interés e inflación en el mercado bursátil español. Tesis doctoral. Departamento de Análisis Económico y Finanzas. UCLM (Albacete).

Jareño, F. (2006b). Sensibilidad de los rendimientos sectoriales a tipos de interés reales e inflación. Investigaciones Económicas, 30(3), 577-610.

Jareño, F. (2007, mayo/junio). Cambio de metodología en la elaboración del IPC y su impacto sobre la respuesta sectorial ante anuncios de inflación. Información Comercial Española, Revista de Economía, 836, 127-140.

Jareño, F. (2008). Spanish Stock Market Sensitivity to Real Interest and Inflation Rates. An Extension of the Stone Two-Factor Model with Factors of the Fama and French Three-Factor Model. Applied Economics, 40(24), 3159-3171.

Jareño, F. (2009). El impacto de la publicación del IPC sobre el mercado bursátil español. Información Comercial Española, Revista de Economía, 851, 109-120.

Jareño, F. \& Navarro, E. (2010). Stock interest rate risk and inflation shocks. European Journal of Operational Research, 201, 337-348.

Jareño, F. \& Tolentino, M. (2012a, November). Inflation Risk Management in Spanish Companies. Archives Des Sciences Journal, 65(11), 10-18.

Jareño, F. \& Tolentino, M. (2012b, November). The Fisher Effect in the Spanish Case: a Preliminary Study. Asian Economic and Financial Review, 2(7), 841-857.

Leibowitz, M. L. \& Kogelman, S. (1990). Inside the P/E Ratio: The Franchise Factor. Financial Analysts Journal, 46(6), 17-35.

Leibowitz, M. L. \& Kogelman, S. (1991). The Franchise Factor for Leveraged Firms. Financial Analysts Journal, 47(6), 29-43.

Leibowitz, M. L. \& Kogelman, S. (1993). Resolving the Equity Duration Paradox. Financial Analysts Journal, 49(1), 51-64.

Leibowitz, M. L. \& Kogelman, S. (2000). Spread-Driven Dividend Discount Models. Financial Analysts Journal, 56(6), 64-81.

Limpanithiwat, K. \& Rungsombudpornkul, L. (2010). Relationship between Inflation and Stock Prices in Thailand. Master thesis in Finance. Umeå School of Business. Spring semester 2010.

Veronesi, P. (1999). Stock market overreaction to bad news in good times: a rational expectations equilibrium model. The Review of Financial Studies, 12(5), 975-1007. 